\title{
Factors associated with the 6-minute walk distance in patients with systemic sclerosis
}

\section{(1) $\operatorname{coseshatx}^{2}$}

Sébastien Sanges 1,2,3,4,5, Jonathan Giovannelli ${ }^{1,2,3,4,5}$, Vincent Sobanski ${ }^{1,2,3,4,5}$, Sandrine Morell-Dubois ${ }^{3,4,5}$, Hélène Maillard ${ }^{3,4,5}$, Marc Lambert ${ }^{1,2,4,5}$, Céline Podevin ${ }^{3,4}$, Nicolas Lamblin ${ }^{6}$, Pascal De Groote ${ }^{6}$, Jean-François Bervar ${ }^{7}$, Thierry Perez ${ }^{8}$, Régis Matran ${ }^{8}$, Martine Rémy-Jardin ${ }^{9}$, Pierre-Yves Hatron 1,2,3,4,5, Éric Hachulla, ${ }^{1,2,3,4,5}$ and David Launay ${ }^{1,2,3,4,5^{*}}$

\begin{abstract}
Background: There is an ongoing debate regarding the relevance of the 6-minute walking distance (6MWD) in systemic sclerosis (SSC) assessment, widely used as a usual test in these patients as well as an outcome measure in clinical trials. In this work, we aimed to assess the associations between the 6MWD and various disease parameters in patients with SSC.

Methods: Consecutive patients followed in our SSC National Reference Centre were included in this cross-sectional study if they fulfilled the 2013 American College of Rheumatology/European League Against Rheumatism criteria for SSc. Data were systematically collected during a comprehensive standardized evaluation that included a 6-minute walk test, clinical assessment, biological results, pulmonary function tests, transthoracic echocardiography, composite scores (European Scleroderma Study Group Activity Index, Medsger severity score, Health Assessment Questionnaire-Disability Index (HAQ-DI)) and treatments.

Associations of the 6MWD with various disease parameters were assessed by linear regression in univariate and multivariate analyses.

Results: The study population comprised 298 patients (females 81\%; mean age $58.2 \pm 13.3$ years; limited cutaneous SSc 82\%; interstitial lung disease (ILD) 42\%; pulmonary arterial hypertension (PAH) 6\%). The 6MWD was significantly and independently associated with gender, age, body mass index, baseline heart rate (HR), HR variation during the test, PAH, history of arterial thrombosis and C-reactive protein levels, as well as with the HAQ-DI score in a sensitivity analysis. Muscle involvement, joint involvement and ILD were not independently associated with the 6MWD.

Conclusions: During SSC, the 6MWD is independently associated with initial HR and HR variation; with PAH but not $I L D$, suggesting that pulmonary vasculopathy may have a greater impact than parenchymal involvement on functional limitation; and with global markers of disease activity and patient disability. These results give clinicians further insight into how to interpret the 6MWD in the context of SSC.
\end{abstract}

Keywords: Systemic sclerosis, 6-minute walk test, Chronotropic incompetence, Exercise tolerance, Interstitial lung disease, Pulmonary hypertension

\footnotetext{
* Correspondence: david.launay@univ-lille2.fr

${ }^{1}$ University of Lille, INSERM U995, LIRIC — Lille Inflammation Research

International Center, F-59000 Lille, France

${ }^{2}$ INSERM U995, F-59000 Lille, France

Full list of author information is available at the end of the article
} 


\section{Background}

Systemic sclerosis (SSc) is a severe condition classified within the connective tissue disease spectrum [1]. Over the last decades, the respiratory complications of the disease, namely pulmonary hypertension $(\mathrm{PH})$ and interstitial lung disease (ILD), have become the leading cause of mortality among SSc patients [2]. One of the most important objectives when managing SSc patients is therefore to detect these complications as early as possible, to properly assess their severity and to accurately follow up therapeutic efficacy.

Among the different tools available to evaluate SSc patients, the 6-minute walk test (6MWT) is simple, noninvasive, inexpensive and reproducible, and is frequently used in daily practice [3]. Initially developed to study exercise tolerance in aviation personnel [4], its use has since been translated to the field of cardiopulmonary diseases [5], such as chronic obstructive pulmonary disease (COPD), idiopathic pulmonary fibrosis (IPF) and idiopathic pulmonary arterial hypertension (PAH). In these mono-organ conditions, the 6-minute walk distance (6MWD) has proven to be an accurate reflection of disease severity with a prognostic significance [6-8].

However, conversely to these diseases, SSc is a systemic condition associated with various extrapulmonary features (skin fibrosis, musculoskeletal pain, heart involvement, anemia) and with significant disability that can confound the interpretation of the 6MWT. In this multi-organ setting, the assumption that the 6MWD is an adequate surrogate marker for the severity of cardiopulmonary complications is no longer straightforward, and this has led clinicians to wonder what is actually being measured during this test in SSc [9]. This is of crucial importance, since the 6MWD is used in daily practice to follow up SSc patients [3], and as an outcome measure in clinical trials for SSc-PAH [10-13] and ILD [14].

Several teams have tried to answer this question but their results are conflicting [15-30]. For instance, forced vital capacity (FVC) measured during pulmonary function tests (PFT) was significantly associated with the $6 \mathrm{MWD}$ in some studies $[19,21,22,25]$ but not in others $[16,18,20,28,29]$. Similarly, associations with musculoskeletal parameters have been observed inconsistently $[21,25,26]$.

To address this issue, we performed a cross-sectional study and assessed the associations between the 6MWD and various disease characteristics in a large and wellphenotyped SSc patient population.

\section{Methods}

\section{Patient selection}

We designed a cross-sectional study and recruited consecutive patients over 18 years old followed in the National Referral Centre for Systemic Sclerosis of Lille from November 2014 to August 2016. They were included in the study if they fulfilled the 2013 ACR/ EULAR classification criteria for SSc [31]. There was no exclusion criterion.

\section{Data collection}

Data were collected systematically by a physician in a standardized case-report form for all patients referred to our day-patient clinic. All of them underwent a comprehensive evaluation performed within the same day (except for transthoracic echocardiography (TTE) that could be performed up to 6 months before or after the 6MWT).

Patients underwent a non-encouraged 6MWT as recommended [32]. The total 6MWD (in absolute and relative values [33]), modified Borg score, peripheral oxygen saturation $\left(\mathrm{SpO}_{2}\right)$, blood pressure (BP) and heart rate $(\mathrm{HR})$ were recorded at the beginning and the end of the test. Variations of $(\Delta)$ Borg score, $\mathrm{SpO}_{2}, \mathrm{BP}$ and $\mathrm{HR}$ were defined as the difference between their final and initial values.

Other data collected included patient and SSc characteristics, clinical parameters (including modified Rodnan skin score (mRSS) and New York Heart Association (NYHA) functional class), biological results (including $\mathrm{C}$-reactive protein (CRP), Nt-pro-BNP and creatinin kinase (CK) levels), TTE (including estimated systolic pulmonary artery pressure (sPAP) and left ventricle ejection fraction (LVEF)), PFT (including FVC, total lung capacity (TLC) and diffusing capacity of the lung for carbon monoxide (DLCO)), composite scores (European Scleroderma Study Group Activity Index (EScSG-AI) [34], Medsger severity score [35], Health Assessment Questionnaire-Disability Index (HAQ-DI) [36]) and treatments (for a complete list of collected data, see Additional file 1).

\section{Statistical analyses}

Characteristics of the population were described using the mean \pm standard deviation (SD) for quantitative variables, and number (percentage) for qualitative variables.

In order to study the associations between the 6MWD (in absolute value) as the dependent variable and the various explanatory variables, in a first step we conducted univariate analyses using linear regressions. Results were expressed using nonadjusted coefficients $(95 \%$ confidence interval (CI)) expressed in meters. To better identify relevant explanatory variables, linear regressions were then adjusted on the main potential confounding factors (gender, age, body mass index (BMI), disease duration, smoking history and SSc subtype).

As a second step, we built a multivariate linear regression model using the following strategy. Firstly, we included a priori the six potential confounding variables 
already described. Secondly, we made a first selection of variables based on univariate analyses $(p<0.10)$. Thirdly, we ensured that among these variables, those of interest were present (i.e., the complications identified a priori as being able to influence the 6MWD: in particular PAH, ILD, joint and muscle symptoms, heart involvement using the left ventricle ejection fraction). Fourthly, we excluded the variables that mediated the relationship between the 6MWD and organ involvements (e.g., symptoms of dyspnea for PAH or ILD), so as not to attenuate the regression coefficients related to organ involvement variables. We also excluded collinear variables and composite scores. Fifthly, we ensured that the number of variables included in the model was consistent with the number of patients (i.e., close to one variable per 10 patients). Finally, we tested several potential interactions: between PAH or ILD and joint symptoms, muscle symptoms, smoking history or SSc subtype; and between $\Delta H R$, initial HR and chronotropic drugs. None of the interactions tested was significant. Additionally, the HAQDI score was included in a separate regression model as a sensitivity analysis. Coefficients (95\% CI) were expressed in meters. Regression diagnostics were performed.

All statistical analyses were performed using $\mathrm{R}$ software, version 3.2.5 [37]. The threshold for statistical significance was set to $p<0.05$.

\section{Results}

\section{Patient characteristics}

Overall, the study population comprised 298 patients (Table 1). Most of them were middle-aged females (sex ratio male/female 0.23 ; mean age $58.2 \pm 13.3$ years) with limited cutaneous SSc (82.2\%) associated with anticentromere antibodies (56.0\%). ILD (41.1\%) and digital ulcers $(43.0 \%)$ were the most frequent complications of the disease, while PAH (5.9\%) and renal crisis (0.4\%) remained rare. Patients were mainly staged in NYHA class I or II (78.7\%) and displayed no major PFT alterations (mean FVC $103.5 \pm 21.8 \%$; mean DLCO $70.0 \pm$ 19.7\%). Musculoskeletal complaints included joint (42.2\%) and muscle symptoms (19.2\%), with mostly normal muscle enzymes (mean CK levels $93 \pm 59.5 \mathrm{IU}$ ).

During the 6MWT, patients walked a mean distance of $438.3 \pm 108.1 \mathrm{~m}(81.6 \pm 17.8 \%$ of the predicted value), decreased their $\mathrm{SpO}_{2}$ by a mean of $0.58 \pm 2.72 \%$ and increased their HR by a mean of $10.4 \pm 11.2 \mathrm{bpm}$. The mean Borg score was $0.4 \pm 1.1$ at the beginning of the test and rose to $2.5 \pm 2.2$ at the end.

\section{Associations with the 6-minute walk distance: nonad- justed and adjusted analyses}

Univariate analyses revealed several associations between the 6MWD and the collected parameters (Table 2). To better identify relevant explanatory variables, these analyses were then adjusted on several potential confounding factors (gender, age, BMI, disease duration, smoking history and SSc subtype).

Regarding general patient and SSc characteristics, the 6MWD was associated with gender $\left(p<10^{-3}\right)$, age $\left(p<10^{-6}\right)$ and BMI $\left(p<10^{-6}\right)$ both before and after adjustment. Associations were observed with smoking habits and disease duration in nonadjusted analysis, but did not remain significant after adjustment. Although we found no association with cutaneous subtype, immunological profile or capillaroscopic anomalies in nonadjusted analysis, adjustment on confounding variables revealed a significant association of the 6MWD with the cutaneous subtype $(p=0.008)$.

Regarding cardiopulmonary parameters, the 6MWD was significantly associated with PAH $\left(p<10^{-6}\right)$, ILD ( $p$ $=0.02$ ) and history of cardiovascular events (venous thrombosis $p=0.001$; arterial thrombosis $p<10^{-3}$ ) in nonadjusted analysis. Association with ILD was no longer significant after adjustment $(p=0.08)$. We also found significant associations of the 6WMD with NYHA functional class $\left(p<10^{-6}\right)$, elevated Nt-pro-BNP levels $\left(p<10^{-6}\right)$, echocardiographic markers of $\mathrm{PH}$ (estimated sPAP $p<10^{-3}$; right atrium area $p=0.01$ ) and left-heart dysfunction (LVEF $p=0.03$ ), and PFT results (TLC $p=0.007$; DLCO $p<10^{-3}$ ) in nonadjusted analysis. These associations remained significant after adjustment.

Other cardiopulmonary parameters measured during the 6MWT also displayed significant associations with the total distance, especially initial $\operatorname{HR}\left(p<10^{-3}\right)$ and $\Delta \mathrm{HR}\left(p<10^{-3}\right)$, both before and after adjustment. Significant associations with chronotropic drug intake were observed only in nonadjusted analysis.

Regarding extracardiopulmonary parameters, we observed an association with articular involvement (joint symptoms $p$ $=0.02$ ) that became nonsignificant after adjustment on confounding variables. Similarly, there was no association with markers of muscular (muscle symptoms $p=0.08$; CK levels $p=0.11$ ) and skin (mRSS $p=0.74$; digital ulcers $p=0.30$ ) involvements in nonadjusted analysis.

Regarding global indicators of disease activity and severity, the 6MWD was significantly associated with hemoglobin $\left(p<10^{-3}\right)$, CRP $\left(p<10^{-3}\right)$, EScSG$\mathrm{AI}(p=0.001)$ and Medsger severity scores $\left(p<10^{-3}\right)$, both before and after adjustment. We also found a markedly large association of the 6MWD with the HAQ-DI score $\left(p<10^{-3}\right)$ in both analyses.

\section{Associations with the 6-minute walk distance: multivariate models}

Based on the results of our nonadjusted and adjusted analyses, we built a multivariate model to determine the factors independently associated with the 6MWD (Table 3). 
Table 1 Characteristics of the study population

\begin{tabular}{llll}
\hline & N & Value \\
\hline Demographic data & & & \\
Females, $n(\%)$ & 298 & 242 & $(81.2)$ \\
Age at inclusion (years), mean (SD) & 298 & 58.2 & $(13.3)$ \\
BMI (kg/m²), mean (SD) & 283 & 24.9 & $(5.3)$ \\
Smoking history, $n$ (\%) & 297 & 119 & $(40.1)$
\end{tabular}

Diagnosis of SSC

$$
\begin{aligned}
& \text { SSc subtype } \\
& \text { ICSSc, } n(\%)
\end{aligned}
$$$$
\text { dcSSc, } n(\%)
$$

Disease duration at inclusion

Since diagnosis (years), mean (SD) (years), mean (SD)

Since Raynaud phenomenon onset (years), mean (SD)

Immunological profile

$$
\begin{aligned}
& \text { Anti-centromere antibodies, } n(\%) \\
& \text { Anti-topoisomerase I antibodies, } n(\%) \\
& \text { Anti-RNA polymerase III antibodies, } n(\%) \\
& \text { Anti-RNP antibodies, } n(\%) \\
& \text { Nailfold capillaroscopy } \\
& \text { Specific organic microangiopathy, } n \text { (\%) }
\end{aligned}
$$

History of organ involvement

Interstitial lung disease

No ILD, $n(\%)$
Limited ILD, $n(\%)$
Extensive ILD, $n(\%)$

Pulmonary arterial hypertension, $n$ (\%)

Scleroderma renal crisis, $n(\%)$

Digital ulcers (previously or at inclusion), $n$ (\%)

Acute venous thrombosis, $n(\%)$

Acute arterial thrombosis, $n$ (\%)

Chronic cardiovascular disease, $n$ (\%)

Clinical evaluation at inclusion

Modified Rodnan skin score, mean (SD)

Telangiectasia, $n$ (\%)

NYHA functional class

Class I, $n(\%)$
Class II, $n(\%)$
Class III, $n(\%)$
Class IV, $n(\%)$

Cardiovascular symptoms, $n$ (\%)

Joint symptoms, $n$ (\%)

Muscle symptoms, $n$ (\%)

6-minute walk test

Total distance $(m)$, mean (SD)

Total distance (\% predicted), mean (SD)

Initial $\mathrm{SpO}_{2}(\%)$, mean (SD)

Final $\mathrm{SpO}_{2}(\%)$, mean (SD)

$\triangle \mathrm{SpO}_{2}(\%)$, mean (SD)

Initial Borg score, mean (SD)

Final Borg score, mean (SD)
Table 1 Characteristics of the study population (Continued)

\begin{tabular}{llll}
\hline & $N$ & Value & \\
\hline$\Delta$ Borg score, mean (SD) & 293 & 2.1 & $(1.9)$ \\
Initial sBP (mmHg), mean (SD) & 292 & 123.8 & $(16.6)$ \\
Final sBP (mmHg), mean (SD) & 292 & 132.9 & $(18.2)$ \\
$\Delta s B P(m m H g)$, mean (SD) & 291 & 9.2 & $(14.0)$ \\
Initial dBP (mmHg), mean (SD) & 271 & 97.4 & $(3.4)$ \\
Final dBP (mmHg), mean (SD) & 292 & 73.6 & $(10.0)$ \\
$\Delta \mathrm{dBP}(\mathrm{mmHg})$, mean (SD) & 291 & 0.95 & $(10.2)$ \\
Initial HR (bpm), mean (SD) & 289 & 76.7 & $(12.3)$ \\
Final HR (bpm), mean (SD) & 288 & 87.1 & $(15.9)$ \\
$\Delta \mathrm{HR}(\mathrm{bpm})$, mean (SD) & 288 & 10.4 & $(11.2)$ \\
Biological data & & & \\
Hemoglobin (g/dl), mean (SD) & 291 & 13.4 & $(1.4)$ \\
ESR (mm/h), mean (SD) & 258 & 14.8 & $(15.3)$ \\
CRP (mg/L), mean (SD) & 293 & 4.0 & $(4.7)$ \\
Creatinin (mg/L), mean (SD) & 294 & 8.0 & $(2.8)$ \\
Estimated GFR (ml/min/1.73 m²), mean (SD) & 294 & 89.1 & $(21.5)$ \\
Elevated Nt-pro-BNP, $n(\%)$ & 290 & 43 & $(14.8)$ \\
CK (IU), mean (SD) & 289 & 93 & $(59.5)$ \\
Ferritin (ng/ml), mean (SD) & 268 & 105.5 & $(136.4)$ \\
Complement activation, $n$ (\%) & 9 & $(3.4)$
\end{tabular}

Transthoracic echocardiography

Left ventricular ejection fraction (\%), mean (SD) $\quad 250$

Left ventricular diastolic dysfunction, $n$ (\%)

Valvular heart disease, $n(\%)$

Peak TRV $(\mathrm{m} / \mathrm{s})$, mean (SD)

RA-RV pressure gradient $(\mathrm{mmHg})$, mean (SD)

Estimated sPAP ( $\mathrm{mmHg}$ ), mean (SD)

RA area $\left(\mathrm{cm}^{2}\right)$, mean (SD)

IVC dilation and/or decreased IVC collapse, $n$ (\%)

Pericardial effusion, $n$ (\%)

Pulmonary function tests

TLC (\% predicted), mean (SD)

FVC (\% predicted), mean (SD)

FEV1 (\% predicted), mean (SD)

FEV1/FVC (\%), mean (SD)

DLCO (\% predicted), mean (SD)

KCO (\% predicted), mean (SD)

$229 \quad 124 \quad(54.2)$

$244 \quad 41 \quad$ (16.8)

$98 \quad 2.62 \quad(0.54)$

$206 \quad 27.4 \quad$ (13.4)

$194 \quad 31.5 \quad$ (13.1)

$139 \quad 15.0$

$210 \quad 16 \quad$ (7.6)

$230 \quad 5 \quad$ (2.2)

$276 \quad 96.2 \quad$ (16.4)

$287 \quad 103.5 \quad$ (21.8)

$286 \quad 95.1 \quad$ (21.8)

$286 \quad 79.2 \quad(10.7)$

$285 \quad 70.0 \quad$ (19.7)

$278 \quad 80.9 \quad$ (17.6)

Composite scores

EScSG-Al score, mean (SD)

Medsger severity score, mean (SD)

HAQ-DI score, mean (SD)

$289 \quad 1.16 \quad(1.2)$

$228 \quad 4.17 \quad(2.5)$

$223 \quad 0.61 \quad(0.7)$

Treatments

Negative chronotropic drugs, $n(\%)$

$293 \quad 87$

(29.7)

Positive chronotropic drugs, $n$ (\%)

293

$B M I$ body mass index, $C K$ creatin kinase, $C R P$ C-reactive protein, $d B P$ diastolic blood pressure, $d c$ diffuse cutaneous, DLCO diffusing capacity of the lung for carbon monoxide, ESCSG-Al European Scleroderma Study Group Activity Index, ESR erythrocyte sedimentation rate, FEV1 forced expiratory volume during the first second, FVC forced vital capacity, GFR glomerular filtration rate, HAQ-DI Health Assessment Questionnaire-Disability Index, HR heart rate, ILD interstitial lung disease, $I U$ international unit, $I V C$ inferior vena cava, $K C O$ diffusing coefficient for carbon monoxide, $l c$ limited cutaneous, NYHA New York Heart Association, $R A$ right atrium, $R V$ right ventricle, $S B P$ systolic blood pressure, $S D$ standard deviation, $S P A P$ systolic pulmonary arterial ventricle, $S B P$ systolic blood pressure, $S D$ standard deviation, $S P A P$ systolic pulmonary arterial
pressure, $\mathrm{SpO}_{2}$ peripheral oxygen saturation, $S S C$ systemic sclerosis, $T L C$ total lung capacity, $T R V$ tricuspid regurgitation velocity, $\Delta$ variation of 
Table 2 Nonadjusted and adjusted associations between 6MWD and SSC characteristics

\begin{tabular}{|c|c|c|c|c|c|c|}
\hline & Nonadj & & & Adjuste & & \\
\hline & $\bar{\beta}$ & $(95 \% \mathrm{Cl})$ & $p$ & $\beta$ & $(95 \% \mathrm{Cl})$ & $p$ \\
\hline Male (vs female) & 52.7 & $(21.8 ; 83.6)$ & $<10^{-3}$ & 76.9 & $(46.4 ; 107.4)$ & $<10^{-3}$ \\
\hline Age (per 1-year increment) & -3.6 & $(-4.5 ;-2.8)$ & $<10^{-6}$ & -3.9 & $(-4.8 ;-3.1)$ & $<10^{-6}$ \\
\hline Smoking history (vs no history) & 31.8 & $(6.9 ; 56.7)$ & 0.01 & -11.2 & $(-34.9 ; 12.6)$ & 0.36 \\
\hline BMI (per $1-\mathrm{kg} / \mathrm{m}^{2}$ increment) & -4.1 & $(-6.4 ;-1.7)$ & $<10^{-3}$ & -3.7 & $(-5.7 ;-1.6)$ & $<10^{-3}$ \\
\hline dcSSc (vs ICSSc) & -0.7 & $(-32.8 ; 31.5)$ & 0.97 & -40.2 & $(-69.7 ;-10.8)$ & 0.008 \\
\hline Disease duration since diagnosis (per 1-year increment) & -1.8 & $(-3.3 ;-0.3)$ & 0.02 & 0.7 & $(-0.8 ; 2.2)$ & 0.38 \\
\hline $\begin{array}{l}\text { Disease duration since first non-RP symptom (per } \\
\text { 1-year increment) }\end{array}$ & -2.3 & $(-3.8 ;-0.7)$ & 0.004 & -0.7 & $(-2.3 ; 0.8)$ & 0.33 \\
\hline Disease duration since RP onset (per 1-year increment) & -1.2 & $(-2.2 ;-0.2)$ & 0.02 & 0.4 & $(-0.5 ; 1.4)$ & 0.38 \\
\hline$A C A$ (vs no ACA) & -23.4 & $(-48.1 ; 1.3)$ & 0.06 & -9.8 & $(-35.5 ; 16.0)$ & 0.46 \\
\hline ATA (vs no ATA) & 0.1 & $(-31.0 ; 31.3)$ & 0.99 & -2.4 & $(-31.5 ; 26.8)$ & 0.87 \\
\hline ARA (vs no ARA) & 47.2 & $(-33.2 ; 127.7)$ & 0.25 & 14.7 & $(-61.6 ; 90.9)$ & 0.71 \\
\hline Anti-RNP antibodies (vs no anti-RNP antibodies) & 3.7 & $(-52.2 ; 59.5)$ & 0.90 & -22.3 & $(-71.0 ; 26.4)$ & 0.37 \\
\hline Abnormal nailfold capillaroscopy (vs normal) & -18.1 & $(-86.7 ; 50.6)$ & 0.61 & -12.5 & $(-66.9 ; 41.8)$ & 0.65 \\
\hline PAH (vs no PAH) & -131.8 & $(-183.2 ;-80.3)$ & $<10^{-6}$ & -112.0 & $(-155.9 ;-68.2)$ & $<10^{-3}$ \\
\hline ILD (vs no ILD) & & & 0.02 & & & 0.08 \\
\hline Limited ILD & 16.4 & $(-13.0 ; 45.3)$ & & 14.2 & $(-13.8 ; 42.2)$ & \\
\hline Extensive ILD & -52.3 & $(-95.6 ;-9.0)$ & & -40.4 & $(-78.7 ;-2.1)$ & \\
\hline DU (previously or at inclusion) (vs no DU) & 13.2 & $(-11.6 ; 38.0)$ & 0.30 & -10.6 & $(-33.7 ; 12.6)$ & 0.37 \\
\hline History of venous thrombosis (vs no history) & -65.0 & $(-103.4 ;-26.5)$ & 0.001 & -52.5 & $(-87.3 ;-17.6)$ & 0.003 \\
\hline History of arterial thrombosis (vs no history) & -73.6 & $(-109.0 ;-38.1)$ & $<10^{-3}$ & -43.5 & $(-76.4 ;-10.6)$ & 0.01 \\
\hline History of cardiovascular disease (vs no history) & -103.8 & $(-150.2 ;-57.5)$ & $<10^{-3}$ & -65.0 & $(-108.2 ;-21.8)$ & 0.003 \\
\hline Modified Rodnan skin score (per 1-point increment) & -0.4 & $(-2.4 ; 1.7)$ & 0.74 & -0.9 & $(-3.1 ; 1.3)$ & 0.42 \\
\hline Telangiectasia (vs no telangiectasia) & -12.4 & $(-41.0 ; 16.1)$ & 0.39 & 13.4 & $(-12.6 ; 39.4)$ & 0.31 \\
\hline NYHA functional class (vs class I) & & & $<10^{-6}$ & & & $<10^{-6}$ \\
\hline NYHA class II & -76.1 & $(-100.1 ;-52.2)$ & & -61.7 & $(-83.8 ;-39.6)$ & \\
\hline NYHA class III & -132.1 & $(-160.8 ;-103.3)$ & & -108.1 & $(-134.5 ;-81.7)$ & \\
\hline NYHA class IV & -227.4 & $(-303.1 ;-151.7)$ & & -216.0 & $(-281.6 ;-150.4$ & \\
\hline Cardiovascular symptoms (vs no symptoms) & -49.8 & $(-94.6 ;-4.9)$ & 0.03 & -33.5 & $(-73.3 ; 6.2)$ & 0.10 \\
\hline Joint symptoms (vs no symptoms) & -29.4 & $(-54.3 ;-4.6)$ & 0.02 & -5.8 & $(-28.4 ; 16.8)$ & 0.61 \\
\hline Muscle symptoms (vs no symptoms) & -27.8 & $(-58.9 ; 3.4)$ & 0.08 & -16.1 & $(-44.0 ; 11.8)$ & 0.26 \\
\hline Initial $\mathrm{SpO}_{2}$ (per $1 \%$ increment) & 14.9 & $(9.2 ; 20.7)$ & $<10^{-6}$ & 11.4 & $(6.3 ; 16.4)$ & $<10^{-3}$ \\
\hline Final $\mathrm{SpO}_{2}$ (per $1 \%$ increment) & 9.1 & $(5.5 ; 12.8)$ & $<10^{-3}$ & 7.2 & $(3.9 ; 10.4)$ & $<10^{-3}$ \\
\hline$\Delta \mathrm{SpO}_{2}$ (per $1 \%$ increment) & 4.7 & $(0.0 ; 9.4)$ & 0.05 & 3.7 & $(-0.3 ; 7.8)$ & 0.07 \\
\hline Initial Borg score (per 1-point increment) & -22.5 & $(-33.3 ;-11.7)$ & $<10^{-3}$ & -21.5 & $(-30.7 ;-12.3)$ & $<10^{-3}$ \\
\hline Final Borg score (per 1-point increment) & -17.7 & $(-23.0 ;-12.4)$ & $<10^{-6}$ & -13.8 & $(-18.6 ;-8.9)$ & $<10^{-6}$ \\
\hline$\Delta$ Borg score (per 1-point increment) & -15.9 & $(-22.1 ;-9.6)$ & $<10^{-3}$ & -10.4 & $(-16.3 ;-4.6)$ & $<10^{-3}$ \\
\hline Initial sBP (per 1-mmHg increment) & -1.0 & $(-1.7 ;-0.2)$ & 0.01 & 0.4 & $(-0.3 ; 1.2)$ & 0.26 \\
\hline Final sBP (per 1-mmHg increment) & -0.6 & $(-1.2 ; 0.1)$ & 0.11 & 0.8 & $(0.1 ; 1.5)$ & 0.02 \\
\hline$\Delta \mathrm{sBP}$ (per 1-mmHg increment) & 0.4 & $(-0.5 ; 1.3)$ & 0.37 & 0.5 & $(-0.2 ; 1.3)$ & 0.18 \\
\hline Initial dBP (per 1-mmHg increment) & 9.1 & $(5.3 ; 12.8)$ & $<10^{-3}$ & 7.1 & $(3.9 ; 10.3)$ & $<10^{-3}$ \\
\hline Final dBP (per 1-mmHg increment) & 0.5 & $(-0.7 ; 1.8)$ & 0.42 & 0.1 & $(-1.0 ; 1.2)$ & 0.91 \\
\hline$\Delta \mathrm{dBP}$ (per 1-mmHg increment) & 0.6 & $(-0.7 ; 1.8)$ & 0.36 & 0.0 & $(-1.0 ; 1.1)$ & 0.95 \\
\hline
\end{tabular}


Table 2 Nonadjusted and adjusted associations between 6MWD and SSC characteristics (Continued)

\begin{tabular}{|c|c|c|c|c|c|c|}
\hline & Nonadje & & & Adjuste & & \\
\hline & $\beta$ & $(95 \% \mathrm{Cl})$ & $p$ & $\beta$ & $(95 \% \mathrm{Cl})$ & $p$ \\
\hline Initial HR (per 1-bpm increment) & -1.9 & $(-2.9 ;-0.9)$ & $<10^{-3}$ & -2.0 & $(-2.9 ;-1.2)$ & $<10^{-3}$ \\
\hline Final HR (per 1-bpm increment) & -0.1 & $(-0.9 ; 0.7)$ & 0.86 & 0.1 & $(-0.6 ; 0.8)$ & 0.85 \\
\hline$\Delta \mathrm{HR}$ (per 1-bpm increment) & 2.1 & $(1.0 ; 3.2)$ & $<10^{-3}$ & 2.5 & $(1.6 ; 3.5)$ & $<10^{-6}$ \\
\hline Hemoglobin (per 1-g/dl increment) & 26.6 & $(18.0 ; 35.2)$ & $<10^{-6}$ & 19.9 & $(11.5 ; 28.2)$ & $<10^{-3}$ \\
\hline ESR (per 1-mm/h increment) & -2.3 & $(-3.1 ;-1.5)$ & $<10^{-6}$ & -2.1 & $(-2.8 ;-1.3)$ & $<10^{-6}$ \\
\hline CRP (per 1-mg/L increment) & -5.8 & $(-8.4 ;-3.3)$ & $<10^{-3}$ & -5.8 & $(-8.0 ;-3.5)$ & $<10^{-3}$ \\
\hline Creatinin (per 1-mg/L increment) & -3.6 & $(-8.0 ; 0.8)$ & 0.11 & -3.4 & $(-7.3 ; 0.5)$ & 0.09 \\
\hline Estimated GFR (per 1-ml/min/1.73 m² increment) & 1.1 & $(0.6 ; 1.7)$ & $<10^{-3}$ & 0.0 & $(-0.6 ; 0.5)$ & 0.97 \\
\hline Elevated Nt-pro-BNP (vs normal Nt-pro-BNP) & -95.4 & $(-128.7 ;-62.1)$ & $<10^{-6}$ & -66.5 & $(-98.4 ;-34.6)$ & $<10^{-3}$ \\
\hline CK (per 1-IU increment) & 0.2 & $(0.0 ; 0.4)$ & 0.11 & 0.2 & $(0.0 ; 0.3)$ & 0.11 \\
\hline Ferritin (per 1-ng/ml increment) & 0.0 & $(-0.1 ; 0.1)$ & 0.55 & 0.0 & $(-0.1 ; 0.1)$ & 0.84 \\
\hline Complement activation (vs no activation) & 1.6 & $(-70.0 ; 73.3)$ & 0.96 & 10.5 & $(-51.2 ; 72.1)$ & 0.74 \\
\hline LVEF (per 1\% increment) & 2.1 & $(0.2 ; 4.1)$ & 0.03 & 2.1 & $(0.4 ; 3.8)$ & 0.02 \\
\hline LVDD (vs no LVDD) & -69.6 & $(-96.6 ;-42.7)$ & $<10^{-6}$ & -17.1 & $(-45.6 ; 11.4)$ & 0.24 \\
\hline Valvular heart disease (vs no valvular heart disease) & -22.5 & $(-59.3 ; 14.2)$ & 0.23 & 26.2 & $(-7.5 ; 59.9)$ & 0.13 \\
\hline Peak TRV (per 1-m/s increment) & -62.3 & $(-101.0 ;-23.6)$ & 0.002 & -33.2 & $(-67.4 ; 1.1)$ & 0.06 \\
\hline RA-RV pressure gradient (per 1-mmHg increment) & -2.5 & $(-3.6 ;-1.5)$ & $<10^{-3}$ & -1.8 & $(-2.8 ;-0.9)$ & $<10^{-3}$ \\
\hline Estimated sPAP (per 1-mmHg increment) & -2.5 & $(-3.6 ;-1.4)$ & $<10^{-3}$ & -1.9 & $(-2.9 ;-0.9)$ & $<10^{-3}$ \\
\hline RA area (per $1-\mathrm{cm}^{2}$ increment) & -4.7 & $(-8.2 ;-1.2)$ & 0.01 & -4.6 & $(-8.4 ;-0.7)$ & 0.02 \\
\hline Abnormal IVC (vs normal IVC) & -26.0 & $(-79.7 ; 27.6)$ & 0.34 & -22.5 & $(-68.9 ; 24.0)$ & 0.34 \\
\hline Pericardial effusion (vs no pericardial effusion) & -57.4 & $(-154.7 ; 39.9)$ & 0.25 & -95.3 & $(-188.3 ;-2.2)$ & 0.05 \\
\hline TLC (per 1\% increment) & 1.0 & $(0.3 ; 1.8)$ & 0.007 & 1.1 & $(0.4 ; 1.8)$ & 0.002 \\
\hline FVC (per 1\% increment) & 0.4 & $(-0.2 ; 0.9)$ & 0.20 & 1.2 & $(0.7 ; 1.7)$ & $<10^{-3}$ \\
\hline FEV1 (per 1\% increment) & 0.3 & $(-0.3 ; 0.9)$ & 0.29 & 1.0 & $(0.5 ; 1.5)$ & $<10^{-3}$ \\
\hline FEV1/FVC (per 1\% increment) & 0.8 & $(-0.3 ; 2.0)$ & 0.16 & -0.2 & $(-1.3 ; 0.9)$ & 0.68 \\
\hline DLCO (per 1\% increment) & 1.4 & $(0.8 ; 2.0)$ & $<10^{-3}$ & 1.5 & $(1.0 ; 2.0)$ & $<10^{-6}$ \\
\hline KCO (per 1\% increment) & 0.7 & $(0.0 ; 1.5)$ & 0.04 & 1.2 & $(0.6 ; 1.9)$ & $<10^{-3}$ \\
\hline EScSG-Al score (per 1-point increment) & -16.4 & $(-26.2 ;-6.5)$ & 0.001 & -14.6 & $(-23.5 ;-5.7)$ & 0.001 \\
\hline Medsger severity score (per 1-point increment) & -10.8 & $(-16.3 ;-5.4)$ & $<10^{-3}$ & -82.9 & $(-99.8 ;-66.0)$ & $<10^{-6}$ \\
\hline HAQ-DI score (per 1-point increment) & -104.6 & $(-122.0 ;-87.2)$ & $<10^{-6}$ & -11.1 & $(-16.2 ;-6.1)$ & $<10^{-3}$ \\
\hline Positive chronotropic drug intake (vs no intake) & -68.4 & $(-136.5 ;-0.3)$ & 0.05 & -38.7 & $(-98.6 ; 21.1)$ & 0.21 \\
\hline Negative chronotropic drug intake (vs no intake) & -48.1 & $(-74.7 ;-21.4)$ & $<10^{-3}$ & -15.3 & $(-41.0 ; 10.5)$ & 0.25 \\
\hline
\end{tabular}

$\beta$ coefficients expressed in meters

*Analyses adjusted on gender, age, BMI, disease duration, smoking history and SSc subtype

6MWD 6-minute walk distance, ACA anti-centromere antibodies, ARA anti-RNA polymerase III antibodies, ATA anti-topoisomerase I antibodies, BMI body mass index, $C l$ confidence interval, $C K$ creatin kinase, $C R P$ C-reactive protein, $d B P$ diastolic blood pressure, $d c$ diffuse cutaneous, $D L C O$ diffusing capacity of the lung for carbon monoxide, DU digital ulcers, ESCSG-Al European Scleroderma Study Group Activity Index, ESR erythrocyte sedimentation rate, FEV1 forced expiratory volume during the first second, FVC forced vital capacity, GFR glomerular filtration rate, HAQ-DI Health Assessment Questionnaire-Disability Index, HR heart rate, ILD interstitial lung disease, $I U$ international unit, IVC inferior vena cava, KCO diffusing coefficient for carbon monoxide, Ic limited cutaneous, LVDD left ventricular diastolic dysfunction, $L V E F$ left ventricle ejection fraction, NYHA New York Heart Association, $P A H$ pulmonary arterial hypertension, $R A$ right atrium, $R P$ Raynaud phenomenon, $R V$ right ventricle, $s B P$ systolic blood pressure, $S P A P$ systolic pulmonary arterial pressure, $\mathrm{SpO}_{2}$ peripheral oxygen saturation, $S S C$ systemic sclerosis, $T L C$ total lung capacity, $T R V$ tricuspid regurgitation velocity, $\triangle$ variation of

Parameters that were significantly and independently associated with 6MWD were gender $(\beta=58.9(22.6$; 95.2) $\mathrm{m}$ for men vs women, $p=0.002)$, age $(\beta=-2.8$ $(-3.9 ;-1.7) \mathrm{m}$ per 1 -year increment, $\left.p<10^{-3}\right)$, BMI $(\beta$ $=-2.7(-5.1 ;-0.2) \mathrm{m}$ per $1-\mathrm{kg} / \mathrm{m}^{2}$ increment, $\left.p=0.03\right)$, history of arterial thrombosis $(\beta=-39.6(-77.9 ;-1.4) \mathrm{m}$ vs no history, $p=0.04)$, PAH $(\beta=-79.2(-129.7 ;-28.8)$ $\mathrm{m}$ vs no PAH, $p=0.002)$, CRP $(\beta=-4.3(-6.7 ;-1.9) \mathrm{m}$ per $1-\mathrm{mg} / \mathrm{L}$ increment, $\left.p<10^{-3}\right)$, initial HR $(\beta=-1.1$ $(-2.2 ;-0.1) \mathrm{m}$ per 1 -bpm increment, $p=0.03)$ and $\Delta \mathrm{HR}$ 
Table 3 Multivariate regression model assessing the associations between 6MWD and SSc characteristics

\begin{tabular}{|c|c|c|c|}
\hline & $\beta$ & $(95 \% \mathrm{Cl})$ & $p$ \\
\hline Male (vs female) & 58.9 & $(22.6 ; 95.2)$ & 0.002 \\
\hline Age (per 1-year increment) & -2.8 & $(-3.9 ;-1.7)$ & $<10^{-3}$ \\
\hline Disease duration since diagnosis (per 1-year increment) & -0.7 & $(-2.5 ; 1.0)$ & 0.42 \\
\hline BMI (per $1-\mathrm{kg} / \mathrm{m}^{2}$ increment) & -2.7 & $(-5.1 ;-0.2)$ & 0.03 \\
\hline Smoking history (vs no history) & -8.2 & $(-33.9 ; 17.5)$ & 0.53 \\
\hline History of venous thrombosis (vs no history) & -10.8 & $(-48.6 ; 26.9)$ & 0.57 \\
\hline History of arterial thrombosis (vs no history) & -39.6 & $(-77.9 ;-1.4)$ & 0.04 \\
\hline dcSSc (vs IcSSc) & -22.7 & $(-57.6 ; 12.2)$ & 0.20 \\
\hline PAH (vs no PAH) & -79.2 & $(-129.7 ;-28.8)$ & 0.002 \\
\hline ILD (vs no ILD) & & & 0.15 \\
\hline Limited ILD & 22.0 & $(-6.9 ; 50.9)$ & \\
\hline Extensive ILD & -14.7 & $(-55.8 ; 26.4)$ & \\
\hline Joint symptoms (vs no symptoms) & -16.5 & $(-41.6 ; 8.6)$ & 0.20 \\
\hline Muscle symptoms (vs no symptoms) & -7.5 & $(-40.6 ; 25.7)$ & 0.66 \\
\hline Hemoglobin (per 1-g/dl increment) & 9.2 & $(-0.8 ; 19.1)$ & 0.07 \\
\hline CRP (per 1-mg/L increment) & -4.3 & $(-6.7 ;-1.9)$ & $<10^{-3}$ \\
\hline Estimated GFR (per 1-ml/min/1.73 $\mathrm{m}^{2}$ increment) & 0.2 & $(-0.4 ; 0.9)$ & 0.46 \\
\hline Initial HR (per 1-bpm increment) & -1.1 & $(-2.2 ;-0.1)$ & 0.03 \\
\hline$\Delta H R$ (per 1-bpm increment) & 2.8 & $(1.8 ; 3.8)$ & $<10^{-6}$ \\
\hline LVEF (per 1\% increment) & 1.2 & $(-0.5 ; 2.9)$ & 0.15 \\
\hline Positive chronotropic drug intake (vs no intake) & 14.2 & $(-44.3 ; 72.6)$ & 0.63 \\
\hline Negative chronotropic drug intake (vs no intake) & -25.3 & $(-52.3 ; 1.7)$ & 0.07 \\
\hline
\end{tabular}

$N=192 . R^{2}=0.59$, adjusted $R^{2}=0.54$

$\beta$ coefficients expressed in meters

6MWD 6-minute walk distance, $B M I$ body mass index, $C l$ confidence interval, $C R P$ C-reactive protein, $d c$ diffuse cutaneous, GFR glomerular filtration rate, $H R$ heart rate, ILD interstitial lung disease, Ic limited cutaneous, LVEF left ventricle ejection fraction, PAH pulmonary arterial hypertension, SSC systemic sclerosis, $\triangle$ variation of

$\left(\beta=2.8(1.8 ; 3.8) \mathrm{m}\right.$ per 1 -bpm increment, $\left.p<10^{-6}\right)$ (Fig. 1). This model accounted for $59 \%$ of the variance of the 6MWD.

A sensitivity analysis including the HAQ-DI score revealed its independent association with the 6MWD $(\beta=-77.1(-101.3 ;-53.0) \mathrm{m}$ per 1 -unit increment, $p<10^{-6}$ ) (Additional file 2).

\section{Discussion}

In this cross-sectional study, we tried to identify the factors associated with the 6MWD in a large and fully characterized population of SSc patients. Our results can be summarized as follows: aside from demographic parameters, we observed significant and independent associations of the $6 \mathrm{MWD}$ with $\triangle \mathrm{HR}$ and resting $\mathrm{HR}$, with PAH and history of arterial thrombosis, and with CRP levels; we found no independent association of the 6MWD with ILD or musculoskeletal involvement; and in a sensitivity analysis, the HAQ-DI score was also independently associated with the 6MWD.
Although literature data are conflicting, the results of our univariate analyses mostly confirm findings from previous works, which observed associations of the 6MWD with age, BMI and cardiopulmonary parameters (especially PFT and TTE), but no major influence of musculoskeletal involvement [15-30]. Multivariate analyses from previous studies have also established age [16], gender [18], HAQ scores [21] and CRP [19] as independent factors associated with the 6MWD. Independent associations with dyspnea [16], initial and final $\mathrm{SpO}_{2}$ [18], initial Borg score [18] and DLCO [21, 28] were also reported, but since our goal was to identify associations with specific organ involvements rather than their mediators, we chose not to include these variables in our multivariate model.

\section{Association of the 6MWD with $\triangle \mathrm{HR}$}

Association between the 6MWD and $\triangle \mathrm{HR}$ has never been studied previously in SSc. Interestingly, similar findings were made in IPF [38] and PAH [39] patients, where the variation in HR was found to be an important 

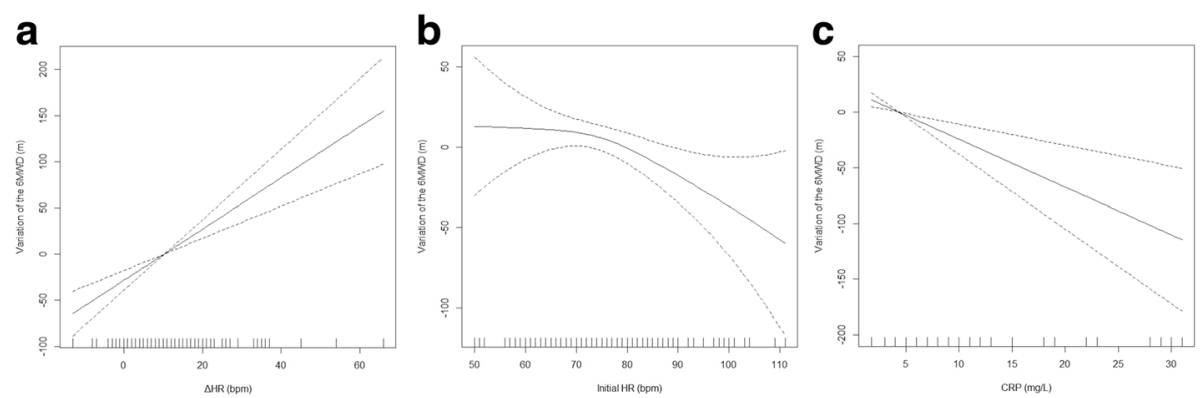

Fig. 1 Adjusted associations in a generalized additive model. Regression splines representing the adjusted associations in a generalized additive model between the 6-minute walk distance (6MWD) and (a) variation of heart rate $(\Delta H R)$ during the test, (b) initial heart rate (HR) at the beginning of the test and (c) C-reactive protein (CRP) levels. y axis corresponds to variation of 6MWD as a function of the explanatory variables. Dashed lines correspond to 95\% Cl of the spline

factor associated with the 6MWD as well as a valuable prognostic marker.

Interpretation of this result in the context of the $6 \mathrm{MWT}$ is challenging. On the one hand, the lower increase in HR observed in some SSc patients could simply indicate lower exercise intensity due to limited functional capacities or a lack of motivation [40]. On the other hand, it could also reflect an actual impairment of the chronotropic response to exercise. Chronotropic incompetence, defined as the inability to increase HR above $80 \%$ of its predicted peak value during a maximal exercise test, is a major cause of exercise intolerance in various cardiopulmonary diseases $[40,41]$. It is a common finding in patients with heart diseases (sick sinus syndrome, atrial fibrillation, ischemic heart disease, chronic heart failure), neurological disorders (through autonomic dysfunction) or specific medication intakes (such as $\beta$-blockers and nondihydropyridine calciumchannel blockers) $[40,41]$.

Since the $6 \mathrm{MWT}$ is a submaximal exercise test, the diagnosis of chronotropic incompetence cannot be made here with certainty; and since detailed patient comorbidities are not available, the causes for the impaired chronotropic response in our population cannot be investigated fully. However, it is interesting to note that negative chronotropic drug intake was not independently associated with the 6MWD in our patients, making the hypothesis of a medication-induced chronotropic incompetence unlikely. Conversely, SSc is frequently associated with autonomic dysfunction [42] (especially in terms of HR regulation [43]) and with a specific myocardial disease [1], both of which could induce chronotropic incompetence.

Overall, this result suggests that an impaired chronotropic response could be an important factor associated with exercise intolerance in SSc. Interestingly, Someya et al. [44] showed in a previous work that the HR at the end of the $6 \mathrm{MWT}$ was higher in SSc patients than in controls to try and compensate for a decreased stroke volume. Thus, it seems reasonable to assume this compensation mechanism would be impaired in SSc patients with chronotropic incompetence, resulting in lower exercise capacities. In any case, this finding warrants further investigations, using maximal incremental exercise tests.

\section{Association of the 6MWD with resting HR}

Interestingly, we found that both $\triangle \mathrm{HR}$ and resting $\mathrm{HR}$ are independently associated with the 6MWD. This result suggests that, during SSc, exercise capacities are conditioned not only by the patient's ability to increase his or her HR during exercise, but also by his or her baseline HR value.

Only two studies have so far tested the association of the 6MWD with resting HR in SSc and they yielded discrepant results $[19,29]$. Our observation is supported by similar findings in patients with nonidiopathic PAH and chronic heart failure [39, 45].

Here again, interpretation of this result in the context of our study is difficult. Heart rate is tightly regulated by the autonomous system in order to adapt cardiac output to situations of stress or exercise [41]. Thus, the reduction of physical performance in SSc patients with increased resting $\mathrm{HR}$ could be suggestive of advanced autonomic dysfunction (loss of balance between sympathetic and vagal basal tones) and/or severe myocardial disease (compensating mechanism for a decreased resting stroke volume), as implied by our previous result. Alternatively, this could also indicate global deconditioning or performance anxiety.

Either way, further studies are needed to better appreciate the pathophysiological implications of this result.

\section{Association of the 6MWD with PAH but not with ILD}

Our multivariate analysis also revealed a significant and independent association of the 6MWD with PAH, but not with ILD. Interestingly, all previous studies that tested the relation between the $6 \mathrm{MWD}$ and $\mathrm{PH}$ consistently found a 
shorter walked distance in $\mathrm{PH}$ patients $[21,30]$ or an association with echocardiographic markers of $\mathrm{PH}[16,19,24,29]$ (with only one exception [18]). Conversely, data regarding the influence of ILD on the 6MWD are more conflicting, with some studies observing shorter 6MWD in SSc-ILD patients $[16,30]$ and others reporting no significant difference $[20,21]$. However, accurate comparison with previous works is challenging because of heterogeneity in design and population (notably in $\mathrm{PH}$ diagnosis, ILD definition and severity).

It is interesting to note that our result is in line with several data pleading for a predominant importance of pulmonary hemodynamic alterations over lung parenchymal involvement. In patients with IPF and $\mathrm{PH}$, the 6MWD is significantly associated with hemodynamic parameters measured during right heart catheterization (RHC), but not with lung volumes assessed by spirometry [46]. In SSc-ILD patients, gas transfer alteration has a stronger prognostic value than lung volume reduction $[47,48]$.

However, if this result confirms that PAH contributes to impaired exercise capacities during SSc, we also observed in a previous work that the $6 \mathrm{MWD}$ correlated poorly with RHC parameters in SSc-PAH patients [49]. Taken together, these results suggest an impact of confounding extracardiopulmonary factors on the walked distance.

\section{Association of the 6MWD with HAQ-DI score}

Another interesting finding of our study is the strong association observed between the 6MWD and the HAQDI score.

Several teams have already reported similar results, both in univariate and multivariate regressions [21, 23]. Remarkably, in a previous work, Chow et al. [50] showed that the HAQ-DI score was not associated with parameters of cardiopulmonary severity in SSc patients with PAH. This implies that the patient perception of disability and functional limitation may not be entirely explained by the actual severity of the disease. Moreover, the 6MWD has been shown to be associated with scales of depression, anxiety and quality of life in several cardiorespiratory diseases $[45,51]$.

Overall, these results suggest that if the 6MWD is influenced to some extent by the objective severity of organ involvements, it is also greatly conditioned by the patient's subjective impression of disability and quality of life impairment.

\section{Association of the 6MWD with CRP}

An independent association between CRP levels and the 6WWD was observed in our population. A similar result was also found in previous work by Schoindre et al. [19] both in univariate and multivariate regressions, but not by Deuschle et al. [21]. Interestingly, CRP levels are associated with the EScSG-AI score, Medgser severity score and HAQ-DI score and with poorer prognosis in SSc patients [52]. In line with our prior results, this observation could suggest that the 6MWD also captures the overall disease activity and severity.

\section{No major influence of the musculoskeletal involvement on the 6MWD}

Contrary to a common idea [9], it is interesting to note that musculoskeletal involvement is not independently associated with the 6MWD in our population. However, we did not consider which sites were involved in patients with joint or muscle symptoms. Therefore, we could not test the association between the 6MWD and lower limb involvement, which could contribute to explain this negative result. The effect of joint and muscle symptoms has been suggested previously in univariate analyses $[21,25,26]$ but has never been tested in multivariate regression. Interestingly, quadricipital strength and joint involvement have been found to correlate both with the 6MWD and with the HAQ-DI score in SSc patients $[25,26,53]$, which suggests that this score may also account for the musculoskeletal-induced disability impacting on the 6MWD.

Our study draws strength from a large sample size and an important number of variables collected.

Our study also has some limitations. We did not perform incremental exercise testing to correlate the 6MWD with objective physiological parameters collected during effort. Similarly, we did not study autonomic dysfunction to help explain the results observed with $H R$ variation and $\Delta H R$.

In our multivariate regression, we chose to include only parameters relating to organ involvement (e.g., PAH and ILD) and exclude variables mediating them (e.g., NYHA class, PFT results and TTE data). Indeed, we believed that inserting the latter in our model could mask interesting associations of the 6MWD with organ involvement.

\section{Conclusion}

Our work showed that, aside from demographic parameters, $\triangle H R$, resting HR, PAH, history of arterial thrombosis, CRP levels and HAQ-DI score are important factors associated with the 6MWD in SSc, while pulmonary interstitial and musculoskeletal involvements seem to have no major influence. This suggests that the 6MWD should be interpreted not only as a marker of cardiorespiratory severity, but also as a global assessment of disease activity and patient disability. Further studies are warranted to investigate the possibility of a chronotropic incompetence as a cause for exercise intolerance in SSc patients. 


\section{Additional files}

Additional file 1: presents additional methods, a complete list of data collected during the study and a definition of each collected item (DOCX 39 kb)

Additional file 2: is Table S1. presenting results of sensitivity analysis with inclusion of the HAQ-DI score in our multivariate regression model assessing the associations between 6MWD and SSc characteristics (DOCX $33 \mathrm{~kb}$ )

\section{Abbreviations}

6MWD: 6-minute walk distance; 6MWT: 6-minute walk test; ACR: American College of Rheumatology; BMI: Body mass index; BP: Blood pressure; CK: Creatinin kinase; COPD: Chronic obstructive pulmonary disease; CRP: Creactive protein; DLCO: Diffusing capacity of the lung for carbon monoxide: EScSG-Al: European Scleroderma Study Group Activity Index;

EULAR: European League Against Rheumatism; FVC: Forced vital capacity; HAQ-DI: Health Assessment Questionnaire_Disability Index; HR: Heart rate: ILD: Interstitial lung disease; IPF: Idiopathic pulmonary fibrosis; LVEF: Left ventricle ejection fraction; mRSS: Modified Rodnan skin score; NYHA: New York Heart Association; PAH: Pulmonary arterial hypertension; PFT: Pulmonary function tests; $\mathrm{PH}$ : Pulmonary hypertension; RHC: Right heart catheterization; SPAP: Systolic pulmonary artery pressure; $\mathrm{SpO}_{2}$ : Peripheral oxygen saturation; SSc: Systemic sclerosis; TLC: Total lung capacity; TTE: Transthoracic echocardiography; $\Delta$ : Variation of

\section{Acknowledgements}

None.

\section{Funding}

None.

\section{Availability of data and materials}

The datasets used and/or analyzed during the current study are available from the corresponding author on reasonable request.

\section{Authors' contributions}

SS, VS and CP acquired patients' data. JG performed all statistical analyses. SS, $J G$ and DL interpreted results and were major contributors in writing the manuscript. NL, PDG, J-FB, TP, RM and MR-J provided cardiorespiratory data and expertise. SM-D, HM, ML, P-YH and EH performed a critical reading of the manuscript. All authors read the final draft of the manuscript and approved its submitted version.

\section{Ethics approval and consent to participate}

The study complied with the recommendations of the Helsinki declaration. French legislation on noninterventional studies does not require ethics committee approval for the use of de-identified data collected during patient care. As such, the need for an ethics committee approval was waived for this study by the "Comité de Protection des Personnes" (CPP).

The data were de-identified and complied with the requirements of the "Commission Nationale de l'Informatique et des Libertés" (CNIL), the organization responsible for ensuring the ethical use of data collected for scientific purposes in France. The CNIL approved the methods used to collect and analyze data from our patient database (approval \#DC-2008-642). Written information was provided to each patient. French legislation on noninterventional studies requires collecting the nonopposition of patients but does not require written consent. As such, nonopposition was obtained from each patient included in the study for the use of their de-identified medical record data.

\section{Consent for publication}

Not applicable.

\section{Competing interests}

The authors declare that they have no competing interests.

\section{Publisher's Note}

Springer Nature remains neutral with regard to jurisdictional claims in published maps and institutional affiliations.

\section{Author details}

University of Lille, INSERM U995, LIRIC — Lille Inflammation Research International Center, F-59000 Lille, France. ${ }^{2}$ INSERM U995, F-59000 Lille, France. ${ }^{3} \mathrm{CHU}$ Lille, Département de Médecine Interne et Immunologie Clinique, F-59000 Lille, France. ${ }^{4}$ Centre National de Référence Maladies Systémiques et Auto-immunes Rares (Sclérodermie Systémique), F-59000 Lille, France. ${ }^{5}$ Health Care Provider of the European Reference Network on Rare Connective Tissue and Musculoskeletal Diseases Network (ReCONNET), F-59000 Lille, France. ${ }^{6} \mathrm{CHU}$ Lille, Service de Cardiologie, F-59000 Lille, France. ${ }^{7} \mathrm{CHU}$ Lille, Service de Pneumologie, F-59000 Lille, France. ${ }^{8} \mathrm{CHU}$ Lille, Service d'Explorations Fonctionnelles Respiratoires, F-59000 Lille, France. ${ }^{9} \mathrm{CHU}$ Lille, Département d'Imagerie Thoracique, F-59000 Lille, France.

Received: 22 September 2017 Accepted: 28 November 2017

Published online: 15 December 2017

\section{References}

1. Hachulla E, Launay D. Diagnosis and classification of systemic sclerosis. Clin Rev Allergy Immunol. 2011;40:78-83.

2. Steen VD, Medsger TA. Changes in causes of death in systemic sclerosis, 1972-2002. Ann Rheum Dis. 2007;66:940-4.

3. Vandecasteele E, De Pauw M, De Keyser F, Decuman S, Deschepper E, Piette $Y$, et al. Six-minute walk test in systemic sclerosis: a systematic review and meta-analysis. Int J Cardiol. 2016:212:265-73.

4. Balke B. A simple field test for the assessment of physical fitness. Rep Civ Aeromed Res Inst US. 1963:6:1-8.

5. Holland AE, Spruit MA, Troosters T, Puhan MA, Pepin V, Saey D, et al. An official European Respiratory Society/American Thoracic Society Technical Standard: field walking tests in chronic respiratory disease. Eur Respir J. 2014;44:1428-46.

6. Miyamoto S, Nagaya N, Satoh T, Kyotani S, Sakamaki F, Fujita M, et al. Clinical correlates and prognostic significance of six-minute walk test in patients with primary pulmonary hypertension: comparison with cardiopulmonary exercise testing. Am J Respir Crit Care Med. 2000; 161:487-92.

7. Lederer DJ, Arcasoy SM, Wilt JS, D'Ovidio F, Sonett JR, Kawut SM. Sixminute-walk distance predicts waiting list survival in idiopathic pulmonary fibrosis. Am J Respir Crit Care Med. 2006;174:659-64.

8. Casanova C, Cote CG, Marin JM, de Torres JP, Aguirre-Jaime A, Mendez R, et al. The 6-min walking distance: long-term follow up in patients with COPD. Eur Respir J. 2007;29:535-40.

9. Impens AJ, Wangkaew S, Seibold JR. The 6-minute walk test in scleroderma-how measuring everything measures nothing. Rheumatol Oxf Engl. 2008:47 Suppl 5:v68-9.

10. Badesch DB, Tapson VF, McGoon MD, Brundage BH, Rubin LJ, Wigley FM, et al. Continuous intravenous epoprostenol for pulmonary hypertension due to the scleroderma spectrum of disease: a randomized, controlled trial. Ann Intern Med. 2000:132:425-34.

11. Rubin LJ, Badesch DB, Barst RJ, Galiè N, Black CM, Keogh A, et al. Bosentan therapy for pulmonary arterial hypertension. N Engl J Med. 2002;346:896-903.

12. Oudiz RJ, Schilz RJ, Barst RJ, Galié N, Rich S, Rubin LJ, et al. Treprostinil, a prostacyclin analogue, in pulmonary arterial hypertension associated with connective tissue disease. Chest. 2004;126:420-7.

13. Ahmadi-Simab K, Hellmich B, Gross WL. Bosentan for severe pulmonary arterial hypertension related to systemic sclerosis with interstitial lung disease. Eur J Clin Invest. 2006:36 Suppl 3:44-8.

14. Seibold JR, Denton CP, Furst DE, Guillevin L, Rubin LJ, Wells A, et al. Randomized, prospective, placebo-controlled trial of bosentan in interstitial lung disease secondary to systemic sclerosis. Arthritis Rheum. 2010;62:2101-8.

15. Buch $M H$, Denton CP, Furst DE, Guillevin L, Rubin LJ, Wells AU, et al. Submaximal exercise testing in the assessment of interstitial lung disease secondary to systemic sclerosis: reproducibility and correlations of the 6min walk test. Ann Rheum Dis. 2007:66:169-73.

16. Villalba WO, Sampaio-Barros PD, Pereira MC, Cerqueira EM, Leme CA, Marques-Neto JF, et al. Six-minute walk test for the evaluation of pulmonary disease severity in scleroderma patients. Chest. 2007;131:217-22.

17. Ciurzyński M, Bienias P, Lichodziejewska B, Szewczyk A, GlińskaWielochowska M, Jankowski K, et al. Assessment of left and right ventricular diastolic function in patients with systemic sclerosis. Kardiol Pol. 2008:66: 269-76. discussion 277-8. 
18. Garin MC, Highland KB, Silver RM, Strange C. Limitations to the 6-minute walk test in interstitial lung disease and pulmonary hypertension in scleroderma. J Rheumatol. 2009;36:330-6.

19. Schoindre Y, Meune C, Dinh-Xuan AT, Avouac J, Kahan A, Allanore Y. Lack of specificity of the 6-minute walk test as an outcome measure for patients with systemic sclerosis. J Rheumatol. 2009;36:1481-5.

20. Akdogan A, Kaya EB, Sahin A, Okutucu S, Yakut E, Kalyoncu U, et al. Relationship between left ventricular diastolic dysfunction and six minute walk test in patients with systemic sclerosis. Int J Rheum Dis. 2011;14:379-83.

21. Deuschle K, Weinert K, Becker MO, Backhaus M, Huscher D, Riemekasten G. Six-minute walk distance as a marker for disability and complaints in patients with systemic sclerosis. Clin Exp Rheumatol. 2011;29:S53-9.

22. Wilsher M, Good N, Hopkins R, Young P, Milne D, Gibson A, et al. The sixminute walk test using forehead oximetry is reliable in the assessment of scleroderma lung disease. Respirology. 2012;17:647-52.

23. Chung L, Chen H, Khanna D, Steen VD. Dyspnea assessment and pulmonary hypertension in patients with systemic sclerosis: utility of the University of California, San Diego, Shortness of Breath Questionnaire. Arthritis Care Res. 2013;65:454-63.

24. Irzyk K, Bienias P, Kostrubiec M, Rymarczyk Z, Bartoszewicz Z, Siwicka M, et al. Six-minute walk test reflects neurohormonal activation and right ventricular function in systemic sclerosis patients. Clin Exp Rheumatol. 2013; 31:18-23.

25. Lima TRL, Guimarães FS, Silva LA, Silva DPG, Menezes SLS, Lopes AJ. Relationship between functional capacity, joint mobility and pulmonary function in patients with systemic sclerosis. J Bodyw Mov Ther. 2015;19:17-24.

26. Lima TRL, Guimarães FS, Carvalho MN, Sousa TLM, Menezes SLS, Lopes AJ. Lower limb muscle strength is associated with functional performance and quality of life in patients with systemic sclerosis. Braz J Phys Ther. 2015;19:129-36.

27. Rizzi M, Sarzi-Puttini P, Airoldi A, Antivalle M, Battellino M, Atzeni F. Performance capacity evaluated using the 6-minute walk test: 5-year results in patients with diffuse systemic sclerosis and initial interstitial lung disease. Clin Exp Rheumatol. 2015;33:S142-7.

28. Lopes AJ, Ferreira Ade S, Lima TRL, Menezes SLS, Guimarães FS. An explanatory model of functional exercise capacity in patients with systemic sclerosis: considerations for rehabilitation programs. J Phys Ther Sci. 2016;28:569-75.

29. Kusunose $K$, Yamada $H$, Nishio $S$, Hirata $Y$, Seno $H$, Saijo $Y$, et al. Echocardiographic Predictors for Worsening of Six-Minute Walk Distances in Patients With Systemic Sclerosis (Scleroderma). Am J Cardiol. 2017:120:315-21.

30. Vandecasteele E, Thevissen K, Melsens K, De Keyser F, De Pauw M,

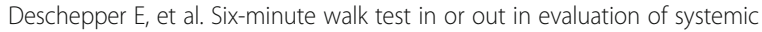
sclerosis patients? Clin Exp Rheumatol. 2017;35 Suppl 106(4):122-129.

31. van den Hoogen F, Khanna D, Fransen J, Johnson SR, Baron M, Tyndall A, et al. 2013 classification criteria for systemic sclerosis: an American College of Rheumatology/European League Against Rheumatism collaborative initiative. Ann Rheum Dis. 2013;72:1747-55.

32. ATS Committee on Proficiency Standards for Clinical Pulmonary Function Laboratories. ATS statement: guidelines for the six-minute walk test. Am J Respir Crit Care Med. 2002;166:111-7.

33. Enright PL, Sherill DL. Reference equations for the six-minute walk in healthy adults. Am J Respir Crit Care Med. 1998;158:1384-7.

34. Valentini G, Rossa AD, Bombardieri S, Bencivelli W, Silman AJ, D’Angelo S, et al. European multicentre study to define disease activity criteria for systemic sclerosis. II. Identification of disease activity variables and development of preliminary activity indexes. Ann Rheum Dis. 2001;60:592-8.

35. Medsger TA, Bombardieri S, Czirjak L, Scorza R, Rossa AD, Bencivelli W. Assessment of disease severity and prognosis in systemic sclerosis. Clin Exp Rheumatol. 2003;21:S42-6.

36. Fries JF, Spitz $P$, Kraines RG, Holman HR. Measurement of patient outcome in arthritis. Arthritis Rheum. 1980;23:137-45.

37. R Development Core Team. R: A language and environment for statistical computing. Vienna, Austria: R Foundation for Statistical Computing; 2008. http://www.R-project.org.

38. Holland AE, Hill CJ, Glaspole I, Goh N, Dowman L, McDonald CF. Impaired chronotropic response to 6-min walk test and reduced survival in interstitial lung disease. Respir Med. 2013;107:1066-72.

39. Provencher S, Chemla D, Hervé $P$, Sitbon O, Humbert M, Simonneau G. Heart rate responses during the 6-minute walk test in pulmonary arteria hypertension. Eur Respir J. 2006;27:114-20.
40. Lauer MS. Heart rate response in stress testing: clinical implications. ACC Curr J Rev. 2001:10:16-9.

41. Brubaker PH, Kitzman DW. Chronotropic incompetence: causes, consequences, and management. Circulation. 2011;123:1010-20.

42. Amaral TN, Peres FA, Lapa AT, Marques-Neto JF, Appenzeller S. Neurologic involvement in scleroderma: a systematic review. Semin Arthritis Rheum. 2013:43:335-47.

43. Bienias P, Ciurzyński M, Glińska-Wielochowska M, Szewczyk A, Korczak D, Kalińska-Bienias $\mathrm{A}$, et al. Heart rate turbulence assessment in systemic sclerosis: the role for the detection of cardiac autonomic nervous system dysfunction. Rheumatol Oxf Engl. 2010;49:355-60.

44. Someya F, Mugii N, Oohata S. Factors relating to impaired stroke volume during the 6-minute walk test in patients with systemic sclerosis. Clin Exp Rheumatol. 2016;34 Suppl 100:152-6.

45. Ingle L, Rigby AS, Nabb S, Jones PK, Clark AL, Cleland JG. Clinical determinants of poor six-minute walk test performance in patients with left ventricular systolic dysfunction and no major structural heart disease. Eur J Heart Fail. 2006:8:321-5.

46. Minai OA, Santacruz JF, Alster JM, Budev MM, McCarthy K. Impact of pulmonary hemodynamics on 6-min walk test in idiopathic pulmonary fibrosis. Respir Med. 2012;106:1613-21.

47. Goh NS, Hoyles RK, Denton CP, Hansell DM, Renzoni EA, Maher TM, et al. Short term pulmonary function trends are predictive of mortality in interstitial lung disease associated with systemic sclerosis: pulmonary function trends in SSC-ILD. Arthritis Rheumatol. Arthritis Rheumatol. 2017; 69(8):1670-1678

48. Le Gouellec N, Duhamel A, Perez T, Hachulla A-L, Sobanski V, Faivre J-B, et al. Predictors of lung function test severity and outcome in systemic sclerosis-associated interstitial lung disease. PLoS One. 2017;12:e0181692.

49. Sanges S, Launay D, Rhee RL, Sitbon O, Hachulla É, Mouthon L, et al. A prospective study of the 6 min walk test as a surrogate marker for haemodynamics in two independent cohorts of treatment-naïve systemic sclerosis-associated pulmonary arterial hypertension. Ann Rheum Dis. 2016; 75:1457-65

50. Chow S, Pope JE, Mehta S. Lack of correlation of the health assessment questionnaire disability index with lung parameters in systemic sclerosis associated pulmonary arterial hypertension. Clin Exp Rheumatol. 2008:26:1012-7.

51. Spruit MA, Watkins ML, Edwards LD, Vestbo J, Calverley PMA, Pinto-Plata V, et al. Determinants of poor 6-min walking distance in patients with COPD: the ECLIPSE cohort. Respir Med. 2010;104:849-57.

52. Muangchan C, Harding S, Khimdas S, Bonner A. Canadian Scleroderma Research Group, Baron M, et al. Association of C-reactive protein with high disease activity in systemic sclerosis: results from the Canadian Scleroderma Research Group. Arthritis Care Res. 2012;64:1405-14.

53. Clements PJ, Wong WK, Hurwitz EL, Furst DE, Mayes M, White B, et al. Correlates of the disability index of the health assessment questionnaire: a measure of functional impairment in systemic sclerosis. Arthritis Rheum. 1999:42:2372-80.

\section{Submit your next manuscript to BioMed Central and we will help you at every step:}

- We accept pre-submission inquiries

- Our selector tool helps you to find the most relevant journal

- We provide round the clock customer support

- Convenient online submission

- Thorough peer review

- Inclusion in PubMed and all major indexing services

- Maximum visibility for your research

Submit your manuscript at www.biomedcentral.com/submit 